\title{
SOME TILINGS OF THE PLANE WHOSE SINGULAR POINTS FORM A PERFECT SET
}

\author{
MARILYN BREEN
}

\begin{abstract}
Let $\mathcal{T}$ be a tiling of the plane such that for every tile $T$ of $\mathcal{T}$ there correspond a tile $T^{\prime}$ of $\mathcal{T}$ (not necessarily unique) and an integer $k\left(T, T^{\prime}\right)$ (depending on $T$ and $\left.T^{\prime}\right), 2<k$, such that $T$ meets $T^{\prime}$ in $k\left(T, T^{\prime}\right)$ connected components. Then the set of singular points of $\mathcal{T}$ is a nowhere dense, perfect set.
\end{abstract}

1. Introduction. We begin with some preliminary definitions. The family $\sigma$ is a tiling for the plane if and only if $\sigma$ is a collection of closed topological disks having pairwise disjoint interiors for which $U\{T: T$ in $\mathscr{T}\}=R^{2}$. Point $p$ in $R^{2}$ is a singular point of $\mathcal{T}$ if and only if every neighborhood of $p$ meets infinitely many tiles of $\mathcal{T}$, and a tiling having no singular point is said to be locally finite. The reader is referred to Grünbaum and Shepard [1] for a thorough treatment of these topics.

In [2], Valette examined tilings $\mathcal{T}$ of the plane having the property that for every tile $T$ of $\mathcal{T}$ there correspond a tile $T^{\prime}$ of $\mathcal{\sigma}$ (not necessarily unique) and an integer $k\left(T, T^{\prime}\right)$ (depending on $T$ and $\left.T^{\prime}\right), 2<k$, such that $T$ meets $T^{\prime}$ in $k\left(T, T^{\prime}\right)$ connected components. While examples reveal that such tilings exist, Valette proved that no such tiling can be locally finite. Here we use his results to obtain the following theorem: For tiling $\mathcal{T}$ having the property above, the set of singular points of $\mathcal{T}$ is a perfect set. That is, the set of singular points of $\sigma$ is closed and has no isolated points. Furthermore, the set is nowhere dense in the plane.

Throughout the paper, bdry $S$ will be used to denote the boundary for set $S$.

\section{The results.}

THEOREM 1. Let $\mathcal{T}$ be a tiling of the plane such that for every tile $T$ of $\mathcal{T}$ there correspond a tile $T^{\prime}$ of $\mathcal{T}$ (not necessarily unique) and an integer $k\left(T, T^{\prime}\right)$ (depending on $T$ and $\left.T^{\prime}\right), 2<k$, such that $T$ meets $T^{\prime}$ in $k\left(T, T^{\prime}\right)$ connected components. Then the set of singular points of $\mathcal{T}$ is a nowhere dense, perfect set.

Proof. The following terminology will be useful. If $T_{1}$ and $T_{2}$ are two associated tiles in $\mathcal{T}$ (that is, tiles which satisfy our hypothesis), let $\left\{D_{12}^{i}\right\}$ be the family of closures of the bounded components of complement $\left(T_{1} \cup T_{2}\right), 1 \leqslant i \leqslant k\left(T_{1}, T_{2}\right)-$ 1 , and let $Y_{12}$ be the closure of the unbounded component. Refer to the collection $\left\{D_{12}^{i}\right\}$ as a bounded component family (of $T_{1}$ and $T_{2}$ ).

From Valette's work, it follows that each $D_{12}^{i}$ is a closed topological disk whose boundary consists of two arcs, one in $T_{1}$ and the other in $T_{2}$, with only their

Received by the editors April 20, 1982 and, in revised form, July 15, 1982.

1980 Mathematics Subject Classification. Primary 52A45. 
endpoints in common. Call such points junction points of set bdry $D_{12}^{i}$. If $D_{12}^{1}$ and $D_{12}^{2}$ intersect, their intersection is a single point which is a junction point of each boundary. No three members of the collection $\left\{D_{12}^{i}, Y_{12}\right\}$ have a point in common. Thus if $D_{34}^{1}$ and $D_{34}^{2}$ are subsets of $D_{12}^{1}$, they cannot intersect at a junction point of bdry $D_{12}^{1}$, since then $D_{34}^{1}, D_{34}^{2}$ and $Y_{34}$ would have a point in common. We will digress to establish the following preliminary result.

LEMMA 1. Let $D$ be a member of any bounded component family. Then $D$ contains infinitely many singular points of $\mathcal{T}$.

Proof of Lemma 1. To reach a contradiction, assume $D$ contains only finitely many singular points of $\mathcal{T}$. Since $\mathcal{T}$ is a tiling of the plane, disk $D$ contains bounded component families, and we let $\left\{D_{12}^{i}\right\}$ be a minimal bounded component family contained in $D$. (That is, $\left\{D_{12}^{i}\right\}$ is a bounded component family in $D$ containing the fewest singular points.) Each $D_{12}^{i}$ contains a bounded component family which in turn must be minimal. Adapting a proof of Valette [2, Proposition 1, V], it is not hard to see that each $D_{12}^{i}$ must contain a singular point. Moreover, by our choice of $\left\{D_{12}^{i}\right\}$, each $D_{12}^{i}$ must contain every singular point in their union, so there can be only one such point. It follows that all the $D_{12}^{i}$ must have a unique singular point $x$ in common, and $x$ is a junction point of each set bdry $D_{12}^{i}$. Moreover, by previous comments, $i=1,2$. Now $D_{12}^{1}$ contains a minimal bounded component family $\left\{D_{34}^{1}, D_{34}^{2}\right\}$, and the sets $D_{34}^{i}$ must intersect in $x$ since $x$ is the only singular point available in $D_{12}^{1}$. But this contradicts the observation above that two such sets cannot intersect at a junction point of bdry $D_{12}^{1}$. Our assumption is false, and Lemma 1 is established.

We are ready to complete the proof of Theorem 1 . We must show that the set of singular points of $\mathcal{T}$ is perfect. Since the set of singular points of any tiling is closed, it suffices to show that there are no isolated singular points.

Suppose on the contrary that $p$ is an isolated singular point of $\mathcal{T}$, and let $(M, p)$ be a closed circular disk at $p$ whose boundary $C$ is free of any other singular points. Now $(M, p)$ intersects an infinite number of tiles in $\mathcal{T}$, and since at most a finite number of these can intersect $C$, there must be an infinite subcollection contained in $(M, p)$. Each member of this subcollection gives rise to a bounded component family, and if any corresponding $D_{i j}^{k}$ were completely contained in $(M, p)$, then by the Lemma there would be an infinite number of singular points in $(M, p)$, impossible.

Thus each corresponding $D_{i j}^{k}$ must meet $C$, and since $C$ meets at most finitely many tiles, there must be a tile $T$ which serves as the associated tile for an infinite number of tiles in $(M, p)$. Thus bdry $T \cap(M, p)$ contains an infinite set of pairwise different points, one from each of this infinite collection of tiles. Hence bdry $T \cap$ $(M, p)$ contains a singular point. This point must be $p$.

Now note that bdry $T \cap(M, p)$ has an infinite number of components. The same is true of bdry $T \cap(N, p)$ where $(N, p) \subseteq(M, p)$. This means bdry $T$ cannot be locally connected at $p$, which is impossible. Our supposition is false, and $\mathcal{T}$ cannot have an isolated singular point. 
Finally, it is not hard to show that the set of singular points of any tiling is nowhere dense in $R^{2}$. This finishes the proof of Theorem 1 .

We remark that the number 2 in Valette's result and the number 2 in Theorem 1 above are best by [2, Figure 5]. However, if the components mentioned in the hypothesis are required to be arcs, then one of these bounds may be lowered, and minor modifications in Valette's proof yield the following analogue.

Corollary to Valette's Proposition 1. If $\mathcal{T}$ is a tiling of the plane such that every tile meets some other tile in a finite number of components, at least 2 of which are arcs, then $\mathcal{T}$ is not locally finite.

It is interesting to observe that such analogues of Lemma 1 and Theorem 1 fail, as Example 1 illustrates.

EXAMPLE 1. Let $C_{1}(r)$ and $C_{2}(r)$ be the closed half-circles of radius $r$ centered at the origin and lying in the upper and lower half-planes, respectively. Let $L$ be the $x$-axis, and let $S=\cup\left\{C_{1}(n) \cup C_{2}(n) \cup C_{1}(1 / n): n \geqslant 1\right\} \cup L$. Define tiling $\mathcal{T}$ to be the collection of closures of components of $R^{2} \sim S$. Then every tile of $\mathcal{T}$ meets another tile of $\mathcal{T}$ in 2 arcs, yet $\mathcal{T}$ has only one singular point, the origin.

We close with an interesting open problem suggested by the referee: Characterize the perfect sets which occur as the set of singular points of tilings of the type considered here.

The author would like to thank the referee for greatly simplifying the proofs of Lemma 1 and Theorem 1.

\section{REFERENCES}

1. B. Grünbaum and G. C. Shepard, Tilings and patterns, Freeman, San Francisco, Calif. (to appear).

2. Alain Valette, Tilings of the plane by topological disks, Geom. Dedicata 11 (1981), 447-454.

Department of Mathematics, University of OKlahoma, Norman, OKLahoma 73019 